\title{
A Note on Two Theorems by Adams and McGee
}

[This is a penultimate draft. Please quote only from the published version: Review of Symbolic Logic Volume 2, Number 3, September 2009, 509-516.]

\begin{abstract}
Three-valued accounts of conditionals frequently promise (a) to conform to the probabilistic view that conditionals are evaluated by conditional probabilities, and (b) to yield a plausible account of compounds of conditionals. However, McGee (1981) shows that probabilistic validity, the conception of validity most naturally associated with the probabilistic view, cannot be characterized by a finite matrix. Adams (1995) indicates a further generalization of this result. Nevertheless, Adams (1986) provides a description of probabilistic validity in three-valued terms by going beyond the standard framework. Yet the language Adams considers is severely restricted: it does not contain compounds of conditionals. Thus, a natural question arises: Is there a plausible three-valued account of compounds of conditionals which agrees with probabilistic validity on the restricted language? In this note, I develop a general framework in which to address this question. The answer will be negative.
\end{abstract}

\section{Introduction}

On the probabilistic view, conditionals are evaluated by conditional probabilities. Given this constraint on their evaluation, one can identify the valid arguments by a natural epistemic condition: an argument is probabilistically valid ( $p$-valid for short) if the uncertainty of the conclusion cannot exceed the sum of the uncertainties of the premises. ${ }^{1}$ However, probabilistic validity is only defined over a language which does not contain compounds of conditionals.

There have been frequent attempts to provide a three-valued semantics for conditionals which is in line with the probabilistic view. ${ }^{2}$ According to such a semantics, conditionals are neither true nor false when the antecedent is false, and they assume the semantic value of the consequent otherwise. This would result in the following (partial) truth-table for the conditional:

$$
\begin{array}{lll}
(\mathrm{A} & \Rightarrow & \mathrm{B}) \\
T & T & T \\
T & F & F \\
F & U & T \\
F & U & F
\end{array}
$$

\footnotetext{
${ }^{1}$ For an extensive treatment and defense of $p$-validity, see Adams (1975).

${ }^{2}$ Milne (1997) gives a historical overview of the various proposals. For a recent defense, see McDermott (1996).
} 
In addition to a semantics compatible with the probabilistic thesis, a threevalued approach promises to account for compounds of conditionals by extending the truth-tables of the connectives to accommodate a third value.

Yet it seemed that probabilistic validity cannot be captured in a manyvalued framework. McGee (1981) showed the following theorem to hold: $p$ validity cannot be characterized by a finite matrix, i.e. one cannot describe $p$-validity as the preservation of a finite set of designated values. Surprisingly, Adams (1986) provided a description of probabilistic validity in broadly threevalued terms by going beyond the standard framework.

Since Adams considers a language which does not contain compounds of conditionals, one question remains open. One may ask whether there is a plausible three-valued account of compounds of conditionals which agrees with probabilistic validity on the restricted language. In this note, I show that this hope is unlikely to be fulfilled. The resources Adams employs to characterize $p$ validity in three-valued terms depend crucially on the restriction of the language he considers.

\section{Results by Adams and McGee}

Some stage setting is needed. As a generalization of the standard framework, I introduce generalized matrices. This enables one to generalize McGee's theorem as indicated in Adams (1995). Moreover, Adams's strategy of escaping McGee's theorem can be captured as well.

As a base language, consider the sentences of a first-order propositional language consisting of an infinite supply of propositional variables $p_{1}, p_{2}, \ldots$, a binary connective $\wedge$, and a unary connective $\neg$. Wherever use is made of other connectives such as $\vee$, they are taken to be defined from $\wedge$ and $\neg$ in the standard way. The sentences of the restricted conditional language are all sentences of the base language together with all sentences of the form $A \Rightarrow B$, where $A$ and $B$ are in the base language. The restricted conditional language is the language within which Adams's logic of conditionals is formulated. Note that it contains neither conjunctions, negations, nor nestings of conditionals. Let us call the extension of the restricted conditional language which is closed under applications of $\wedge, \neg$, and $\Rightarrow$ the unrestricted conditional language. Finally, a conditional language is supposed to be either the restricted or the unrestricted conditional language.

A consequence relation over a language $L$ is taken to be any relation $\models$ between finite sets of sentences and sentences in $L$ which obeys reflexivity, monotonicity, and cut, i.e. which satisfies

(i) $\{\phi\} \models \phi$,

(ii) If $\Sigma \models \phi$, then $\Sigma \cup \Omega \models \phi$, and

(iii) If $\Sigma \models \psi$ and $\Sigma \cup\{\psi\} \models \phi$, then $\Sigma \models \phi$.

A pair $\langle\Sigma, \phi\rangle$ consisting of a set of sentences $\Sigma$ and a sentence $\phi$ within a language $L$ will be called an argument over $L$; its premises are the sentences in 
$\Sigma$, its conclusion is $\phi$. An argument $\langle\Sigma, \phi\rangle$ is valid according to a consequence relation $=$ iff $\Sigma \mid=\phi$; a sentence is a theorem iff the corresponding argument from the empty set of premises is valid.

A generalized matrix for a conditional language is a tuple $\langle N, R, *, \uparrow,-\rangle$ with the following properties:

- $N$ is any set (the set of semantic values),

- $R$ is a relation between sets of semantic values and semantic values (the relation of admissible assignments),

- * and $\uparrow$ are binary operations on $N$, and - is a unary operation on $N$.

A generalized matrix is $n$-valued if $N$ contains exactly $n$ members.

Given a generalized matrix $M=\langle N, R, *, \uparrow,-\rangle$ and a conditional language $L$, a function $g: L \rightarrow N$ is called an assignment (relative to $M$ ) if it is a homomorphism into the algebra of the matrix, i.e. if for all sentences of $L$ : $g(\phi \wedge \psi)=g(\phi) * g(\psi), g(\phi \Rightarrow \psi)=g(\phi) \uparrow g(\psi), g(\neg \phi)=-g(\phi)$. Given a set of sentences $\Sigma, g(\Sigma)$ is the set of semantic values $g(\phi), \phi \in \Sigma$.

Let us say that a generalized matrix $M$ with $R$ as the relation of admissible assignments is characteristic of a consequence relation $\models$ over a conditional language $L$ iff for all finite sets of sentences $\Sigma$ and sentences $\phi: \Sigma \mid=\phi$ just in case $\langle g(\Sigma), g(\phi)\rangle \in R$ for every assignment $g$ (relative to $M$ ). A consequence relation can be characterized by a generalized n-valued matrix if there is a generalized $n$-valued matrix which is characteristic of it. A consequence relation will be called $n$-valued if it can be characterized by a generalized $n$-valued matrix.

The class of consequence relations which can be characterized by a generalized $n$-valued matrix comprises all consequence relations which can be described in terms of possible combinations of finitely many semantic values for the premises and a semantic value for the conclusion. In this sense, the present framework is maximally general. Consequence relations determined by a standard matrix are a special case: given a standard matrix with semantic values $N$ and designated values $D$, define the relation $R$ of admissible assignments as the set of pairs $\langle X, y\rangle$ satisfying $(y \in D)$ if $(X \subseteq D)$. Consequence relations which can be described by a family of standard matrices can be characterized by a generalized matrix as well.

Adams (1995) indicates that McGee's theorem generalizes to all consequence relations having a certain condensation property. Given two propositional variables $p_{i}$ and $p_{j}, i<j$, and a set of sentences $\Gamma$ in which both of them occur, condensing $p_{i}$ and $p_{j}$ means that $p_{i}$ is substituted for $p_{j}$ at every of its occurrences within sentences in $\Gamma$. Further, let us say that a consequence relation $\models$ has the strong condensation property if there is an $n$ such that for all finite arguments $\langle\Sigma, \phi\rangle$ containing at least $n$ propositional variables: if condensing any two propositional variables in $\Sigma \cup\{\phi\}$ results in a valid argument $\left\langle\Sigma^{\prime}, \phi^{\prime}\right\rangle$, then $\Sigma \models \phi$.

As in McGee's (1981) original proof, one can show that the class of consequence relations which can be characterized by an $n$-valued matrix all have the strong condensation property: 
Remark 2.1. Every consequence relation which can be characterized by a generalized n-valued matrix has the strong condensation property.

It will prove useful to say that a consequence relation $\models$ has the weak condensation property if there is an $n$ such that for all finite arguments $\langle\Sigma, \phi\rangle$ containing at least $n$ propositional variables: if condensing any two propositional variables in $\Sigma \cup\{\phi\}$ results in a valid argument $\left\langle\Sigma^{\prime}, \phi^{\prime}\right\rangle$ without turning $\phi^{\prime}$ into a theorem, then $\Sigma \models \phi$. As is clear from the definition, one has the following relation:

Remark 2.2. If a consequence relation has the strong condensation property, then it also has the weak condensation property.

In his original proof, McGee makes use of the fact that $p$-validity does not have the strong condensation property. Inspection of his proof shows that $p$ validity does not even have the weak condensation property. This will be crucial for the discussion of compounds in the next section.

Observation 2.1. p-validity does not have the weak condensation property.

Proof (sketch). Given any $n$, McGee (1981) considers the following argument containing $n+1$ propositional variables:

(1) $p_{0} \Rightarrow\left(\neg p_{1} \wedge \ldots \wedge \neg p_{n}\right)$

(2) $p_{1} \Rightarrow\left(\neg p_{2} \wedge \ldots \wedge \neg p_{n}\right)$

$\vdots$

(n) $p_{n-1} \Rightarrow \neg p_{n}$

$\therefore \quad p_{n} \Rightarrow\left(\neg p_{0} \vee \ldots \vee \neg p_{n}\right)$

This argument is not $p$-valid. But condensing any two variables in it results in a $p$-valid argument. We should note the following: in condensing $p_{i}$ and $p_{j}$, $i<j$, the condensed premise (i) remains essential for the condensed argument (all other premises become inessential): the argument

(i) $p_{i} \Rightarrow\left(\neg p_{i+1} \wedge \ldots \wedge \neg p_{i} \wedge \ldots \wedge \neg p_{n}\right)$

$\therefore p_{n} \Rightarrow\left(\neg p_{0} \vee \ldots \vee \neg p_{i} \vee \ldots \vee \neg p_{i} \vee \ldots \vee \neg p_{n}\right)$

is $p$-valid for all $i$ but its conclusion is not a theorem for any $i$.

Taken together, one can infer a generalization of McGee's original theorem: p-validity cannot be characterized by a generalized n-valued matrix. A very general result.

In the light of McGee's theorem, it is surprising that Adams (1986) found a way of describing $p$-validity in terms of a three-valued notion of validity. He considers assignments over the restricted language of conditionals which are bivalent on the propositional variables and accord otherwise with the classical 
truth-tables for conjunction and negation and the three-valued table for the conditional. Let us call such assignments A-assignments. An $A$-assignment is said to verify a sentence of the language if it assigns to it the value True; it falsifies a sentence if it assigns to it the value False. Moreover, an $A$-assignment confirms a set of sentences (of the restricted language) if it does not assign falsity to any of the sentences and does assign truth to at least one of the sentences. A set $\Sigma$ of premises yields a conclusion $\phi$ iff (i) every assignment which confirms the premises verifies the conclusion and (ii) every assignment which falsifies the conclusion falsifies at least one premise. Based on this, Adams defines a three-valued concept of validity: A finite argument $\langle\Sigma, \phi\rangle$ over the restricted conditional language is said to be $A$-valid iff a subset $\Sigma^{\prime} \subseteq \Sigma$ of its premises yields its conclusion.

Now, the important result about $A$-validity is that it is equivalent to probabilistic validity with respect to the restricted language of conditionals:

Theorem 2.1 (Adams). Let $\langle\Sigma, \phi\rangle$ be a finite argument of the restricted conditional language. Then $\langle\Sigma, \phi\rangle$ is p-valid iff it is A-valid. ${ }^{3}$

Adams escapes McGee's theorem by generalizing the present framework along two dimensions: (a) he characterizes the valid arguments in terms of properties of subsets of the premises, and (b) he restricts the relevant assignments. Firstly, defining validity in terms of properties of subsets of the premises allows Adams to meet the challenge of McGee's proof: in condensing propositional variables within the relevant argument, some premises always become inessential. But in this case, the whole set of condensed premises does not have to yield the conclusion, a subset will do. This somewhat strange feature is already displayed in very simple cases. For instance, the argument from $q \Rightarrow q$ to $r \Rightarrow r$ is $A$-valid because the conclusion is yielded by the empty set of premises, and not because it is yielded by the premise itself. Secondly, considering only $A$ assignments may seem to be irrelevant, but, as one sees on reflection, it ensures that $A$-validity extends classical validity with respect to the base language.

Let us implement the general features of Adams's strategy into the present framework. Take a generalized matrix $M=\langle N, R, \ldots\rangle$ and an assignment $g$ relative to this matrix. Given a subset $N^{\prime} \subseteq N$, let us say that $g$ is $N^{\prime}$-based if it assigns to the propositional variables only values in $N^{\prime}$. Now, say that $M$ is liberally characteristic of a consequence relation $\models$ over a conditional language $L$ if there is a subset $N^{\prime} \subseteq N$ satisfying this condition: an argument $\langle\Sigma, \phi\rangle$ is valid according to $\models$ iff there is $\Sigma^{\prime} \subseteq \Sigma$ such that $\left\langle g\left(\Sigma^{\prime}\right), g(\phi)\right\rangle \in R$ for every $N^{\prime}$-based assignments $g$. Accordingly, a consequence relation can be liberally characterized by a generalized n-valued matrix if there is a generalized $n$-valued matrix which is liberally characteristic of it. Finally, a consequence relation will be called liberally $n$-valued if it can be liberally characterized by an $n$ valued matrix. Adams's theorem can then be seen as showing that $p$-validity is a liberally 3 -valued consequence relation over the restricted language. ${ }^{4}$ Of

\footnotetext{
${ }^{3}$ See Adams (1986: 264). Further comments are given in Adams (1998: ch. 7).

${ }^{4}$ Note that the notion of yielding can only be captured by a generalized 3-valued matrix; it cannot be described by a family of standard matrices.
} 
course, if a consequence relation is $n$-valued, it is also liberally $n$-valued, but as the case of $p$-validity shows, the class of liberally $n$-valued consequence relations properly extends the class of $n$-valued ones.

\section{A Problem with Compounds of Conditionals}

Can one extend Adams's strategy to compounds of conditionals? For instance, one might try to extend $A$-validity to the unrestricted conditional language by extending the truth tables for the connectives. More generally, one may ask whether there is a finite many-valued consequence relation over the unrestricted language which delivers plausible results for compounds of conditionals and which agrees with $p$-validity on the restricted language. As we shall see shortly, however, this question should be answered in the negative. Any such attempt will violate a plausible constraint on the logic of compound conditionals: it will not allow for a classical conjunction in the language, i.e. it will either violate the introduction or the elimination rule for conjunction. Unless one is willing to grant that conditionals give rise to counterexamples to the introduction or the elimination rule for conjunction, there does not seem to be a plausible way of accounting for compounds of conditionals within a finite many-valued framework without violating probabilistic validity.

If one designs a semantic theory for the unrestricted conditional language, can there be any doubt that the conjunction should obey the standard introduction and elimination rules? The burden of proof would be on the side of those who think that it should not. Counterexamples would have to be produced. So far, there is no evidence that conditionals violate the standard rules of conjunction and it is hard to see how an alleged counterexample could possibly be justified as genuine.

The claim presently made is not supposed to contradict what might be called a dismissive approach to the problem of compounds of conditionals. Proponents of the dismissive approach doubt that a semantic theory for the unrestricted language has to be provided, since they feel that conditionals do not embed freely into complex constructions. ${ }^{5}$ Nevertheless, even proponents of the dismissive approach typically grant that conjunctions of conditionals are fine. However, the observation below will be relevant mainly to those accounts which seek to provide a many-valued truth conditional semantics for the unrestricted language.

To get a feeling for the problem, consider the possibility of extending $A$ validity to compounds of conditionals. Furthermore, assume that conjunctions having a conjunct which is neither true nor false are taken to be true or false or neither true nor false depending on whether the other conjunct is true or false or neither true nor false. Consider the inference from $\neg q \vee r$ to $(q \Rightarrow r) \wedge(\neg q \Rightarrow \neg q)$.

\footnotetext{
${ }^{5}$ Proponents of the dismissive approach include Adams (1975: 31f.), Edgington (1995: 280f.), and to some extent Bradley (2002). One might also mention Belnap (1973), who is less dismissive but reckons with the possibility that implication relations between conditional assertions are quite special and can only be captured by a plurality of consequence relations, some of which might even violate the classical rules for conjunction. Note, though, that Belnap (1973) does not engage in the project of giving a truth conditional semantics for conditionals.
} 
As it happens, the premise would yield the conclusion. If the premise is true, one of the conjuncts will always be undefined and the other one true. If the conclusion is false, the only plausible option is that this is because the first conjunct is false, and then the premise would be false as well. But if the conjunction is classical, then $\neg q \vee r$ would imply $q \Rightarrow r$, which it does not according to $p$-validity. ${ }^{6}$

Now to the general case. Let $\wedge$ be the conjunction of the unrestricted conditional language. Let us call it classical with respect to a consequence relation $=$ iff

(i) If $\Gamma \models \phi$ and $\Gamma \models \psi$, then $\Gamma \models \phi \wedge \psi$,

(ii) If $\Gamma \models \phi \wedge \psi$, then $\Gamma \models \phi$ and $\Gamma \models \psi$,

for all sentences $\phi$ and $\psi$, and finite sets of sentences $\Gamma$. Thus, a conjunction is classical if the standard introduction and elimination rules are valid. As is easily seen, one has the following simple fact $(\bigwedge \Gamma$ is the conjunction of the sentences in $\Gamma$ ):

Remark 3.1. Let $\wedge$ be classical with respect to a consequence relation $\models$ over the unrestricted language. Then $\Gamma \models \phi$ iff $\wedge \Gamma \models \phi$ for all sentences $\phi$ and finite sets of sentences $\Gamma$.

So, once a classical conjunction is in place, the question of whether a finite argument is valid reduces to the question of whether a corresponding single premise argument is valid. Interestingly, one can show that given a classical conjunction, even liberally characterized $n$-valued consequence relations must have the weak condensation property:

Observation 3.1. Let $\models$ be a consequence relation over the unrestricted conditional language, and $\wedge$ be classical with respect to $\models$. Assume that $=$ can be liberally characterized by a generalized $n$-valued matrix. Then $\models$ has the weak condensation property.

Proof. Let $\models$ be liberally characterized by the generalized $n$-valued matrix $M=\langle N, R, \ldots\rangle$ and suppose for reductio that $=$ does not have the weak condensation property. Then there is a finite argument $\langle\Sigma, \phi\rangle$ containing at least $n+1$ propositional variables such that (i) condensing any two of them results in a valid argument whose conclusion is not a theorem, but (ii) $\Sigma \not \models \phi$. Given the classicality of the conjunction, (ii) implies that

(1) $\bigwedge \Sigma \not \models \phi$.

Hence, there is an assignment $g$ (possibly based on some subset $N^{\prime} \subseteq N$ - it will not matter) such that $\langle g(\bigwedge \Sigma), g(\phi)\rangle \notin R$. As in McGee's original proof, there are propositional variables $p_{i}$ and $p_{j}$ such that $g\left(p_{i}\right)=g\left(p_{j}\right)$. Now, let the argument $\left\langle\Sigma^{\prime}, \phi^{\prime}\right\rangle$ result from condensing $p_{i}$ and $p_{j}$. It follows then by the properties of an assignment that $g(\bigwedge \Sigma)=g\left(\bigwedge \Sigma^{\prime}\right)$ and $g(\phi)=g\left(\phi^{\prime}\right)$. Hence, by (1), one infers

\footnotetext{
${ }^{6}$ Examples like this motivate a similar rejection of 3 -valued logics for compounds of conditionals in Bradley (2002).
} 
(2) $\left\langle g\left(\bigwedge \Sigma^{\prime}\right), g\left(\phi^{\prime}\right)\right\rangle \notin R$.

Since, by (i), $\phi^{\prime}$ is not a theorem, (2) implies that the argument from $\Lambda \Sigma^{\prime}$ to $\phi^{\prime}$ is not valid. Given the classicality of the conjunction, it follows that $\left\langle\Sigma^{\prime}, \phi^{\prime}\right\rangle$ is not valid either, contradicting (i).

Recall (see Observation 2.1) that $p$-validity does not even have the weak condensation property. In the light of the present observation, this implies the following corollary:

Corollary 3.1. There is no consequence relation over the unrestricted conditional language which can be liberally characterized by a generalized n-valued matrix and which has the following two additional properties: (a) the conjunction is classical, and (b) it agrees with p-validity on the restricted language.

The presence of a classical conjunction in the unrestricted language reduces the question of which arguments are valid to the question of which arguments from a single premise are valid. But for single premise arguments, there are no interesting subclasses of the set of premises: there is only the whole set (containing the single premise) and the empty set. This is why consequence relations which are only liberally characterized by an $n$-valued matrix still have the weak condensation property (which $p$-validity lacks) if the conjunction is classical. So, it becomes clear that Adams's method of escaping McGee's theorem by defining validity in terms of properties of subsets of the set of premises is essentially bound to the restriction of the language he considers. In the presence of a full-blown classical conjunction, it loses its power.

How does the present observation relate to the triviality results? ${ }^{7}$ The triviality results usually start from the assumption that Adams's thesis (i.e. that the probability of a conditional is the corresponding conditional probability) is extended to nested conditionals and that the probabilities obey the standard laws on the unrestricted language. ${ }^{8}$ It should be pointed out that no such assumption is made here. It is neither assumed that Adams's thesis is extended to nested conditionals, nor is the assumption made that probabilities satisfy all the standard laws on the unrestricted language. Thus, even if the probabilistic view is only assumed to apply to the restricted language, the present observation shows that there cannot be a reasonable many-valued semantic underpinning of the unrestricted language which allows for the presence of a classical conjunction.

This poses a dilemma for proponents of a three-valued account of compounds of conditionals which is supposed to conform to the thesis that conditionals are evaluated by conditional probabilities. Either they will not succeed in defining a classical conjunction, or their conception of validity will disagree with $p$-validity

\footnotetext{
${ }^{7}$ For the original triviality results, see Lewis $(1976,1986)$; for a generalized result, see Bradley (1999).

${ }^{8}$ In this way, the triviality results are connected to the problem of compounds of conditionals. Consider sentences $q$ and $r \Rightarrow q$. If the probabilities obey the standard laws, one will find $P(r \Rightarrow q \mid q) \times P(q)=P(q)$, so $q$ would probabilistically imply $q \wedge(r \Rightarrow q)$. But this clashes with a classical conjunction, for $q$ does not probabilistically imply $r \Rightarrow q$.
} 
on the restricted language. In the latter case, they will either classify some arguments as valid even though the uncertainty of the conclusion can exceed the sum of the uncertainties of the premises, or they will classify some arguments as invalid even though it can never happen that the uncertainty-condition is violated. Both options are not very attractive. So, one might suspect that a marriage between three-valued accounts of compounds of conditionals and the probabilistic view of conditionals will always be unhappy. ${ }^{9}$

\footnotetext{
${ }^{9}$ Acknowledgements. This paper has greatly profited from discussion with Benjamin Schnieder and Alexander Steinberg. Special thanks to Dorothy Edgington for extensive comments on various earlier drafts of the present paper and to Nick Haverkamp, whose expertise in non-classical logics has led to significant improvements of the present paper. I would also like to thank three anonymous referees of this journal for their helpful comments. Research for the present paper has been made possible through the generous support of the Deutsche Forschungsgemeinschaft.
} 


\section{References}

[1] Adams, E.W., 1975. The Logic of Conditionals. Reidel: Dordrecht.

[2] Adams, E.W., 1986. On the Logic of High Probability. Journal of Philosophical Logic 15 (3), pp. 255-279.

[3] Adams, E.W., 1995. Remarks on a Theorem of McGee. Journal of Philosophical Logic 24 (4), pp. 343-348.

[4] Adams, E.W., 1998. A Primer of Probability Logic. Stanford: CLSI Publications.

[5] Belnap, N.D.Jr., 1973. Restricted Quantification and Conditional Assertion. In Leblanc, H. (ed.), Truth, Syntax and Modality. Amsterdam/London: North-Holland Publishing Company, pp. 48-75.

[6] Bradley, R., 1999. More Triviality. Journal of Philosophical Logic 28 (2), pp. 129-139.

[7] Bradley, R., 2002. Indicative Conditionals. Erkenntnis 56 (3), pp. 345378.

[8] Edgington, D., 1995. On Conditionals. Mind 104 (414), pp. 235-329.

[9] Lewis, D., 1976. Probabilities of Conditionals and Conditional Probabilities. Philosophical Review 85 (3), pp. 297-315.

[10] Lewis, D., 1986. Probabilities of Conditionals and Conditional Probabilities II. Philosophical Review 95 (4), pp. 581-589.

[11] McDermott, M., 1996. On the Truth Conditions of Certain If-Sentences. Philosophical Review 105 (1), pp. 1-37.

[12] McGee, V., 1981. Finite Matrices and the Logic of Conditionals. Journal of Philosophical Logic 10 (3), pp. 349-351.

[13] Milne, P., 1997. Bruno de Finetti and the Logic of Conditional Events. British Journal for the Philosophy of Science 48 (2), pp. 195-232. 